\title{
Fault Detection of Mobile Bracket with MATLAB
}

\author{
Shruti Lohade \\ BE E\&TC, D.I.E.M.S, \\ A'bad, Maharastra, \\ India
}

\author{
Roshni John \\ BE E\&TC, D.I.E.M.S, \\ A'bad, Maharastra, \\ India
}

\author{
Ekta Bhojwani \\ BE E\&C, D.I.E.M.S, \\ A'bad, Maharastra, \\ India
}

\author{
Abhijeet Chavan \\ (Dept.E\&Tc), \\ D.I.E.M.S, \\ A'bad, Maharastra, \\ India
}

\begin{abstract}
An internal mobile bracket, also known as its metal body, is the main supporting structure of a mobile phone to which all other components are attached, comparable to the skeleton of an organism. The goal of fault detection system is to handle occurring faults in the bodt as in the dimensions, the slots of the small bolts, the camera placement slots, the sensors of the reciever and trmsmitter, the battery connecting slots etc. as all these slots re very small in some may be visibly detected but some may not.
\end{abstract}

Quality control, cost reduction and above all, human and environmental safety are great reasons that stimulate the investments in technologies like automatic inspection. The automatic inspection of connecting lines in the mobile metal body is of special interest, due to the fact that such connecting lines are not visible by the human eye. Visual inspection is one highest cost in metal frame.

The main motive of the fault detection is that it may reduce human efforts mainly, secondly the error will be more precisely detected which would have being missed out by the human or the machine due misplacements. The machine used for this detection may be time consuming, or if in any case the detction is not done after its making, requires large overhead costs and results in high wastage. After manufacturing product; to make decision of rejecting or accepting is taken by measuring quality parameters. To measure quality parameters such as dimensions and features of manufactured product inspection is many a times not done.

To overcome these problems quality control and quality management for sensitive product is feasible by use of image processing techniques. This technique will detect all the fault in the frame, like the connecting lines, the dimensions, bends, cracks, change in positions of parts like minute nuts and bolts, the missed slots etc. This activity completely captures any metal body of any brand then compares it with the ideal frame then checks each and every fault from all the parts. If the body matches with the ideal body using the image processing technique then the product is forwarded for further process. And if the chassis does not match the ideal one then it is rejected.

\section{General Terms}

Edge detection, segmentation, image fusing, and image recognization

\section{Keywords}

MATLAB, controller, LED'S, Cameras, TxRx (Wireless)

\section{INTRODUCTION}

Image processing is one of the most increasing areas now a day. The edge detection process used to simplify the analysis of images by drastically reducing the amount of data to be processed, while at the same time it store useful geometrical information about object. There is certainly a great deal of variety in edge detection applications, but it is felt that many applications share a common set of necessities. These requirements give up a conceptual edge detection problem, the solution of which can be applied in any of the original problem domains. Here in this paper various types of features extraction techniques are discussed.

Any manufacturing industry follows typical cyclic process during manufacturing products. Quality assurance is very important to withstand in market. Measurement and monitoring are very important steps to follow in quality management cycle which gives assurance of quality of manufactured product. Computers can be used for automatic measurement and monitoring of manufactured product. To check quality of manufactured product it is necessary to identify defects in manufactured product to stay away from delivery to customer.

One of the most important operations on digital image is the image fusing technique. The principle of image fusion using wavelets is to merge the wavelet decompositions of the two original images using fusion methods applied to approximations coefficients and details coefficients. The two images must be of the same size and are supposed to be associated with indexed images on a common colormap. Two examples are examined: the first one merges two different images leading to a new image and the second restores an image from two fuzzy versions of an original image.

\section{OBJECTIVES}
a) Less expensive
b) One time investment.
c) Real time fault detection and selection.
d) Less maintenance.
e) Accuracy of product is high

\section{METHODOLOGY}

Our sysytem consists of cameras, controller and wireless transmitter receiver circuit and all are controlled through MATLAB. A single MATLAB command will set the time or capture the real time image and do all the process.

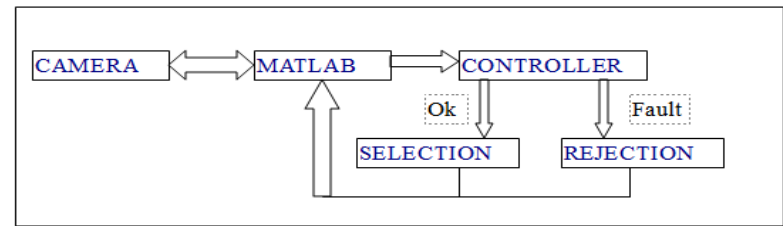

Fig.3.1: System block daigram

The block daigram illustrates that the main components used are Camera, MATLAB sofware and Controller. They functions as follows: 


\subsection{Camera}

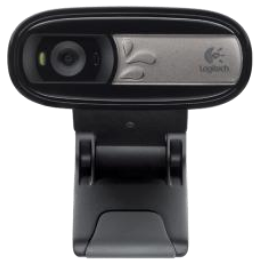

\section{Fig3.1.1: USB camera}

The camera used will capture the image from the top view with appropriate placements and capture the ideal image of the mobile bracket. The second image will be the image which we have to examine i.e the real time image. The real time image which is captured and whih will be captured is all compared to the ideal image for similar type of product.

\subsection{MATLAB}

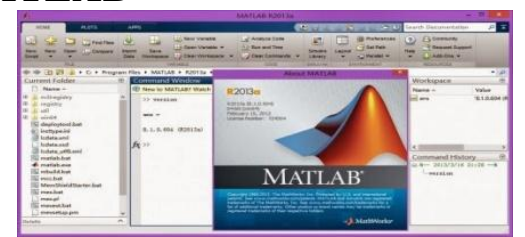

The software which is used is MATLAB. The two images i.e the ideal image and the real time image will be send to the MATLAB through their interfacing. MATLAB will get two types of images one ideal image from the files inside PC and real time image from cameras. After getting both the image, using image processing both the images will be compared. MATLAB will perfom the particular operations as in, the Edge detection, segmentation and mainly then image fusing. The entire real time image compared with their respective ideal image will show the image fused accoring to the results gives the conros to the controller next to it.

\subsection{Controller}

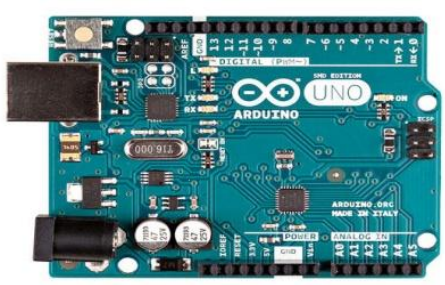

The conroller used is Arduino UNO which will be given the commands by the user by checking both the fused image. If the image is yellow then the user has to press ' $\mathrm{g}$ ' i.e for good and that will be preceded for the further process. But if there exist any other colour other then yellow then it is not perfect and the user has to press ' $\mathrm{f}$ ' i.e for failed and it will be rejected.

\section{SOFTWARE IMPLEMENTATION}

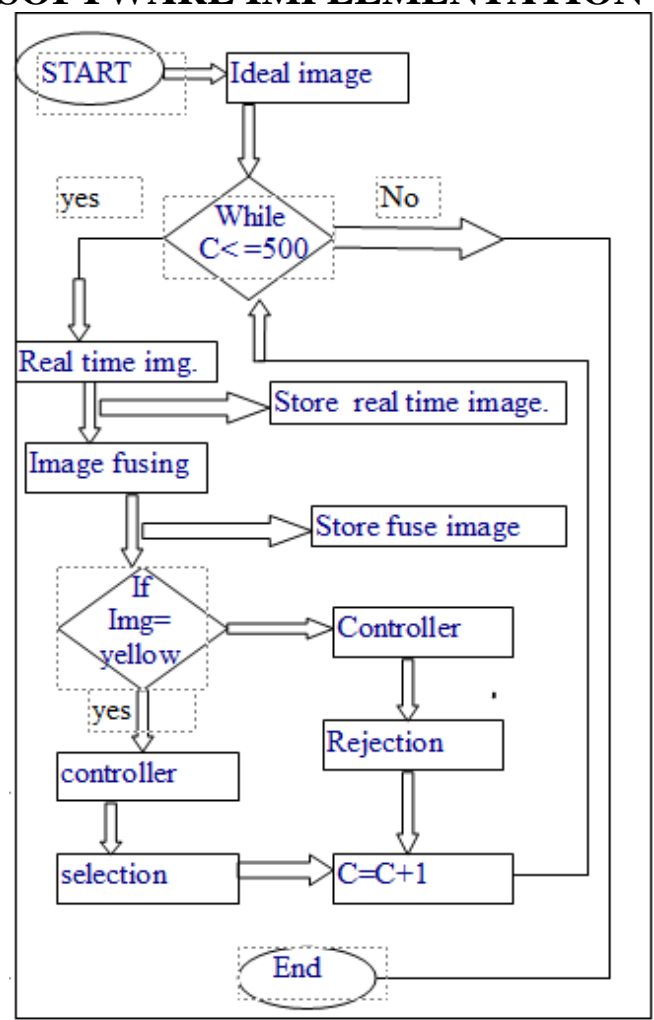

Fig.4.1: The Data Flow of the software

Step 1: The system starts and user sets the ideal image at first. Say ' $C$ ' as the no. of mobile brackets to be detected that can be set by the user according to their production so it can be any figure. Accordingly that such no. of brackets will be examined.

Step 2: The real time image will captured i.e the mobile bracket to detect at that time and it will be stored.

Step 3: The fusing of the real time image and the ideal image is done and if the fused image is complete yellow then it is perfect bracket and send to the controller for its selection process. If the fused image have colour like green or red with the yellow image then the bracket has some parts missing or some parts extra respectively.

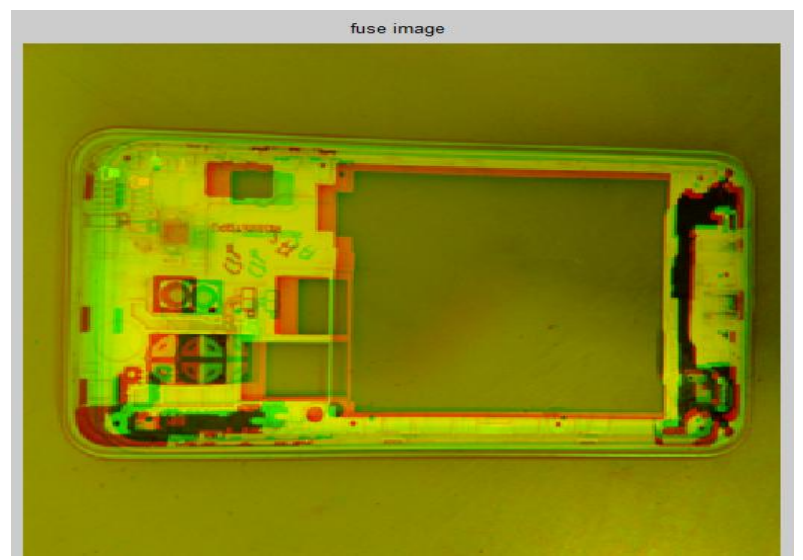

Step 4: The result will be stored with its id number as backup data, and the user just has to press ' $\mathrm{g}$ ' for selection and ' $\mathrm{f}$ ' for rejection through the keyboard which will help in selection of product. 
Step 5: The step 2,3,4 will repeated for the inceasing no. of brackets ' $\mathrm{C}+1$ 'till ' $\mathrm{C}<$ target' and also the results and information will be stored in the system.

\section{RESULT AND ANALYSIS}

Table1: Result classification

\begin{tabular}{|l|l|l|}
\hline $\begin{array}{l}\text { Original and } \\
\text { captured Image }\end{array}$ & Detected image & Analysis \\
\hline & $\begin{array}{l}\text { Red shows } \\
\text { extra and } \\
\text { displaced part } \\
\text { and green } \\
\text { shows missing } \\
\text { part. }\end{array}$ \\
\hline
\end{tabular}

\section{CONCLUSION}

A new era for mobile bracket inspection has started when the electronic imaging systems have gained such a status that it has become possible to take high resolution images. The detected images are saved in computer memory and they can be used to aid the daily operations like production line diagnostic. It is also very important for the system to classify the defects correctly. This can be done with neural networks. With an automatic surface inspection system it is possible to gain a consistent inspection result covering $100 \%$ of the surface. The final task for the system is to judge if the product fits the intended use.This system successfully detects the real time faults in brackets and increases reliability and efficiency of product .It is economically and financially affordable and also reliable.

\section{REFERENCES}

[1] Murthad Al- Yoonus, Mohammed Saeed Jawad, M. F. L.Abdullah and Fares Al-Shargie, "Enhance Quality Control Management for Sensitive Industrial Products Using 2D/3D Image Processing Algorithms," Electrical Power, Electrcs, Communications, Controls, and Informatics Seminar (EECCIS), 2014.

[2] S. Vasilic and Z. Hocenski, "Detecting Methods in Ceramic Defects Detection", In Industrial Electronics, 2006 IEEE International Symposium, vol. 1, pp. 469472, 2006.

[3] Szkilnyk, G., 2012. Vision-based Fault Detection in Assembly Automation. M. A. Sc. Thesis, Queen's University, Kingston, ON.

[4] R. Poli, "Genetic programming for feature detection and image segmentation," The University of Birmingham, School of Computer Science, The University of Birmingham, Edgbaston, Birmingham B 15 2TT, UK, Tech. Rep., 2000. 